ce con alfabeto "ibèrico" de Botorrita, $\mathrm{Za}$ ragoza.

DI: Ho/, J., y MICHLLL:NA, L. (1974): La inscripción celtibérica de Botorrita, Salamanca.

DiAz SANZ, M. A. (1987): Evolución estructural y funcional en el «Cabezo de las Minas» de Botorrita (Zaragoza): siglos ı a. C. a । d. C., I Jornades Internacionals d'Arqueología Romana, 230-235, Museu de Granollers.

Diaz. Sanz, M. A., y Medrano Marquis, M. (1986): Las áreas fabriles de Contrebia Belaisca (Botorrita, Zaragoza): una unidad de producción, Arqueologia Espacial 9, Coloquio sobre el Microespacio 3, 187-207, Teruel.

Diaz Sanz, M. A., y Torralba Martin, J. (1989. 1990): Las ánforas de Contrebia Belaisca: precisiones cronológicas, Caesaraugusta 6667, 29-55, Zaragoza.

Esk^, J. F. (1989): Towards an interpretation of the hispano-celtic inscription of Botorrita, Innsbruck.

FATAS, G. (1980): CONTREBIA BELAISCA (Botorrita. Zaragoza), II, Tabula Contrebiensis, Zaragoza.

Maluquir de Montes, J., et alii (1986): Arquitectura i urhanisme ibèrics a Catalunva, Universidad de Barcelona.

Midirano, M., y Diaz. Sanz, M. A. (1986): Inscripción ibérica sobre vasija tipo «llduradin» hallada en Contrebia Belaisca (Botorrita, Zaragoza), Estudios en Homenaje al Dr. Antonio Beltrán Martinez, 601-611, Zaragoza.

Olmsted, G. (1991): Gaulish, Celtiberian and Indo-European Verse. The Journal of IndoEuropean Studies, Volume 19, n. ${ }^{\circ} 3$ y 4 , 259-307, Virginia.

PAmplona, J. J. (1957): Breve nota de un yacimiento inédito en Botorrita. Caesaraugusta 9-10, 147-150, Zaragoza.

\title{
TIPOS CERÁMICOS Y GEOQUÍMICA: EL CASTRELÍN DE SAN JUAN DE PALUEZAS (LEÓN).
}

POR

\author{
V. GALVÁN*, M. D. FERNÁNDEZ-POSSE**, \\ F.-J. SÁNCHEZ-PALENCIA*** Y J. GALVÁN* \\ ${ }^{*}$ Centro de Ciencias Medioambientales. CSIC. ${ }^{* *}$ ICRBC. \\ Ministerio de Cultura. *** Centro de Estudios Históricos. CSIC
}

RESUMEN

Se discuten algunos métodos de caracterización mineralógica de cerámicas, buscando el establecimiento de tipos que permitan combinar los datos morfométri$\cos$ y la funcionalidad de las vasijas con su estructura fisico-química. Para ello se utilizan varias categorías cerámicas, análisis de fluorescencia de rayos $\mathrm{X}$ y un tratamiento de los datos con análisis multivariante de los óxidos mayoritarios y minoritarios en la composición de la cerámica. Los materiales analizados proceden de castros prerromanos de la Zona Arqueológica de Las Médulas, caracterizados por unas producciones a mano que se engloban dentro de la Cultura Castreña del Noroeste.
SUMMARY

It is discussed some methods of mineralogic characterization of pottery in order to establish a typology that combines morphometric data and the functional character of pottery with its chemical and physical structure. There are used some ceramic groups treated with fluorescence X-rays in which oxides have been analysed. The material used, typical of the Cultura Castre$\tilde{n} a$, is all handmade and come from prerroman sites of the Zona Arqueológica de Las Médulas. 
Este trabajo se integra dentro de un proyecto de investigación a escala regional, Zona Arqueológica de Las Médulas ', que se está llevando a cabo en el Suroeste de León y cuya finalidad última es el conocimiento de los cambios económicos y sociales que experimentaron las sociedades indigenas de la zona al producirse el contacto con el mundo romano. Uno de los factores esenciales de esas transformaciones fue el desarrollo de la minería del oro como nueva actividad a gran escala, planificada y dirigida dentro del marco de los intereses globales romanos en la Peninsula Ibérica. Sin embargo, en el desarrollo de esa investigación hemos tenido ocasión de caracterizar a las comunidades prerromanas astures que ocuparon la zona en los dos siglos anteriores al cambio de era. Estos indigenas prerromanos pertenecen al amplio complejo cultural de la Cultura Castreña del Noroeste. Es decir, su núcleo de población es el castro.

Estos castros se sitúan en emplazamientos muy selectivos de topografía muy regular y constituyen recintos bien delimitados natural y artificialmente. Son de tamaño reducido en comparación con los poblados de otros círculos peninsulares de la Segunda Edad del Hierro -nunca sobrepasan las $2 \mathrm{Ha}$ - - y albergan comunidades también reducidas. Sin embargo, son la unidad básica de ocupación del territorio hasta sus últimas consecuencias sociales. Es decir, cada castro es una comunidad social independiente que desarrolla de forma autárquica una economía de subsistencia de base agropecuaria. Esta falta de cohesión entre los diversos castros tiene su reverso en la organización del espacio interior de cada uno de ellos. En efecto, existe una fuerte interdependencia social y económica entre las unidades de ocupación o viviendas que componen el asentamiento, cuyo carácter cerrado queda aún más de manifiesto en la importancia de los elementos constructivos que lo delimitan - ya sean parapetos, murallas o fosos-, dotados así de un significado

1 El Proyecto de investigación «Zona Arqueológica de las Médulas» está subvencionado por la Junta de Castilla y León y se realiza en el Departamento de Historia Antigua y Arqueología del Centro de Estudios Históricos del CSIC. sociocultural que transciende lo meramente defensivo.

Su patrón de ocupación del territorio es, por lo tanto, autárquico, de la misma forma que su modelo de ocupación de ese territorio es autosuficiente. Es comprensible, pues, que sean comunidades que se mantienen en un relativo aislamiento de otros grupos culturales cercanos. Asi se explica, por ejemplo, que sólo con la presencia romana se introduzca en la zona el molino circular o la cerámica a torno de tipo celtibérico. Sin embargo este menor desarrollo técnico en comparación con las comunidades vecinas de la Meseta y el que su cerámica pertenezca al circulo de la Cultura Castreña gallega, nos ha animado a presentar este trabajo, en la doble razón de que sus resultados son relativamente extrapolables a un ámbito cultural amplio y de que el método para llegar a ellos no carece de novedad.

En tres de estos castros prerromanos leoneses, $\tan$ sucintamente definidos, hemos realizado excavaciones. En dos de ellos en suficiente extensión como para contar con la cantidad de material cerámico necesaria para establecer un repertorio tipológico de sus formas. El primero de ellos es La Corona de Corporales (SánchezPalencia y Fernández-Posse, 1985 y 1986; Fernández-Posse y Sánchez-Palencia, 1988). Está situado en la cima de una colina sobre el curso alto del río Eria, al pie de la vertiente sur de la Sierra del Teleno; muy cerca por tanto de la divisoria entre las cuencas del Duero y del Sil y de uno de los pasos de comunicación entre la Meseta y Galicia. El segundo es El Castrelín de San Juan de Paluezas (Sánchez-Palencia y otros, 1990), sobre la margen izquierda del río Sil. Se emplaza en un espolón definido por dos encajados arroyos desde el que se domina casi todo El Bierzo. Su lado sur, el único relativamente accesible, se prolonga en un paisaje sobre el Sil a la Subfosa de Las Médulas.

En el primero de ellos ya se habían realizado análisis de sus cerámicas (Galván García y Galván Martinez, 1988 y Fernández-Posse y Sánchez-Palencia, 1988: 64-75), aunque sólo con la finalidad de caracterizarlas mineralógicamente y establecer su presumible procedencia local. Para este último objetivo se realiza- 
ron, asimismo, análisis sobre algunas arcillas utilizadas como materia prima y materiales de construcción. Esos estudios sobre la composición mineralógica de las cerámicas de Corporalea fueron realizados en el Instituto de Edafología y Biologia Vegetal del CSIC, hoy en dia Centro de Ciencias Medioambientales y se utilizaron los siguientes métodos: Difracción de Rayos X. Microscopia Electrónica y Espectroscopia Infrarroja, además de su estudio por binocular.

De esta forma se tipificó su homogénea composición mineralógica y quedó clara la autoctonia de la manufactura, que utiliza el material sedimentario más inmediato (muy idóneo por otra parte) decantándolo más o menos según la funcionalidad de cada vasija de aproximadamete $600-700^{\circ}$. Es decir, en aquella ocasión se cumplieron los objetivos citados y quedaron establecidas, además, algunas otras cuestiones referidas principalmente a la estructura física de esas cerámicas que, por otra parte, forman un conjunto homogéneo de indices tipométricos claros que definen un repertorio de formas restringido. Son cerámicas a mano de características técnicas bastante cuidadas como queda de manifiesto en cuestiones como lo fino de sus paredes o en el tratamiento, en ocasiones minucioso, de sus superficies. También es factible la atribución funcional de estas vasijas. Pese a esa homogeneidad general y desde el primer análisis visual de sus componentes físi$\cos$, registramos una característica que parecía significativa: la naturaleza, tamaño y número de desgrasantes eran variables que definian dos «clases» cerámicas. En efecto, las cerámicas más finas, de tamaño reducido y decoradas lo que podriamos denominar desde el punto de vista de su funcionalidad «vajilla»- eran micáceas, es decir, tenian presencia significativa de mica. Las cerámicas de cocina y almacenaje mostraban un aspecto pizarroso.

Esa, en cierto modo, artificial división de las cerámicas en dos grandes grupos por el tipo mayoritario de desgrasante utilizado, perdió parte de su significación cuando se observaron varias muestras de los dos tipos de binocular: su composición no era excesivamente diferente y se habia utilizado en ambas la misma ma- teria prima de pizarras micáceas y de micas moscovitas. El hecho de que a simple vista algunas cerámicas muy determinadas parecieran más micáceas que otras se debia a la orientación de ese material sobre su superficie, lo que les daba un característico aspecto brillante. Y esto se debia simplemente al tratamiento espatulado o bruñido más apurado de su superficie. De todas formas, $y$ aunque todas tenian la misma composición mineralógica - arcillas micáceas-, algunos ejemplares introducían mayor cantidad de micas como desgrasantes, respondiendo, pues, grosso modo a nuestra clasificación visual. Asimismo los resultados de las pruebas por Difracción de Rayos X establecian dos grupos cerámicos con valores bastante diferentes de mica, que en algunos ejemplares llegaban a duplicar el patrón normal. Como esta mayor presencia de mica va unida a un proporcional descenso del cuarzo, parece tratarse de arcillas premeditadamente más depuradas, circunstancia que adquiere significación cuando vemos que se corresponden con las cerámicas voluntariamente más cuidadas y que llevan con frecuencia decoración, contrariamente a las cerámicas más bastas de cocina y almacenaje que presentan siempre los valores más bajos.

Evidentemente el haber realizado esa analitica sobre las cerámicas de Corporales fue una experiencia positiva. Sin embargo no dejaba de ser una solución de corto alcance y cuya validez puede cuestionarse. En primer lugar, porque otorga solamente un valor nominal al contenido de mica, por más que éste permitiera discriminar algunos tipos de pasta. En segundo término, porque muchos fragmentos de cerámica sólo aparentan ese alto nivel de desgrasantes micáceos por el cuidado en el tratamiento de sus superficies, que ha dejado orientadas las brillantes particulas de mica sobre ellas, cuando en realidad el resto de su pasta no contiene valores altos.

En un intento de corregir esas ambigüedades, decidimos un cambio de planteamiento o de estrategia a la hora del estudio de las cerámicas prerromanas de la Zona Arqueológica de Las Médulas, es decir, las del segundo de los yacimientos citados: El Castrelín de San Juan de Paluezas. 
En este sentido consideramos conveniente, pues, comenzar la búsqueda de elementos más concretos y fiables que permitan, además, el establecimiento de verdaderos tipos. $\mathrm{Y}$ todo ello a sabiendas de que el proceso ha de resultar largo y necesita de instrumentos más ajustados como son los análisis cuantitativos de su composición mineralógica y una estadistica más profunda.

Al hablar de tipos no nos referimos solamente a aquéllos que quedan definidos por la tipometria de las cerámicas y su funcionalidad, a lo que preferimos denominar "formas", sino a los resultados de combinar tales tendencias de fabricación con la estructura físico-química y técnica de las cerámicas. Es decir, algo que quizás esté más cerca de lo que podría llamarse "clase» y cuya existencia sea susceptible de quedar demostrada empíricamente. Esto es, una clasificación que resulte, sobre todo, útil a la hora de analizar grandes cantidades de cerámica desde un punto de vista diacrónico.

Entre la cerámica del Castrelín se vislumbran, más que en La Corona, algunas formas con caracteristicas técnicas en apariencia diferentes. Se trata de comprobar si esas diferencias son premeditadas y forman conjuntos individualizados por variables que se refieren tanto a sus componentes y caracteristicas de fabricación, como a su forma y funcionalidad.

En el conjunto bastante homogéneo a simple vista que constituyen las cerámicas de El Castrelín aparecen dos posibles tipos particulares que parecen separarse del común de la cerámica castreña que es como denominaremos al resto de la producción: uno de ellos está compuesto por unas pequeñas ollitas de borde corto $y$ exvasado que tras un cuello marcadamente cilindrico desarrollan un galbo bastante amplio. Pese a su fabricación a mano, presentan un aspecto bastante evolucionado que las separa fácilmente del resto. Poseen paredes muy finas y levantadas con una pasta homogénea, compacta y depurada que produce lineas de roturas regulares y lineales y un característico color gris claro que nos ha determinado a agruparlas bajo la denominación de grises. No son demasiado abundantes en el yacimiento.

El segundo grupo lo constituyen unas vasi- jas no menos caracteristicas que suelen llevar una terminación cepillada o escobillada en su superficie con cierta ambición decorativa. Estas vasijas, que denominaremos grupo escohillado, suelen ser ollas de dimensiones medias y se caracterizan tipológicamente por un cuello marcadamente troncocónico y un desarrollado galbo de perfil globular de tendencia también troncocónica. Su boca, bastante cerrada, termina en un grueso y corto borde. Su aspecto técnico, como su forma, se separa del resto de la cerámica del yacimiento, presentando un aspecto arcilloso y compacto con escasos desgrasantes y frecuente coloración rojiza. Suelen presentar ennegrecidas las superficies exteriores con una concrección carbonosa. La terminación cepillada vertical que les da nombre está regularmente aplicada sobre el cuello y parte superior de la panza de la vasija.

El conjunto restante, el que denominamos cerámica castreña, es mayoritario. Se acomoda a las características morfométricas, dentro de una considerable tendencia a la estandarización, y de fabricación propias de la cerámica castreña del ámbito cultural a que pertenece, es decir, el Noroeste peninsular, con abundantes ejemplos en esta zona astur-leonesa objeto de nuestro estudio: unas producciones de pastas poco depuradas donde predominan los colores oscuros y los tratamientos espatulados.

Además de estos tres conjuntos cerámicos consideramos conveniente hacer objeto del estudio algunos ejemplares de cerámica a torno, del tipo de pasta anaranjada, y de unos característicos moldes de situla. Ambos con el objeto de saber, como era presumible en el caso de los otros conjuntos, si su procedencia era local. La inclusión de estas dos categorias han de entenderse en su contexto, como indicadores que son de la interacción en unas comunidades que hemos presentado como independientes en sus relaciones espaciales y económicas. Sobre todo, este punto de vista es obligado en el caso de los moldes cerámicos de sítulas ya que nos hablan de unos contactos o intercambios en el seno de un ámbito regional extenso, puesto que este tipo de sítula, además de unas características fijas y repetitivas, ofrece una amplia dis- 
persión en castros del occidente gallego y portugués (Carballo, 1983).

Resumiendo, los objetivos de este trabajo son por tanto saber:

- Si existen diferencias geoquímicas entre los conjuntos de cerámicas enumerados más arriba, es decir cerámicas grises, escobilladas, castreñas, a torno y situlas.

- Si esa cerámica a torno y los moldes de situla son o no de fabricación local.

\section{MATERIALES Y MÉTODOS}

Para llevar a cabo nuestros objetivos se seleccionaron 34 muestras de cerámica y arcillas de seis categorias arqueológicas distintas todas ellas de carácter arqueológico: los grupos cerámicos arriba definidos - gris, escobillado, castreño, torno-y otros elementos como moldes de situlas y arcillas utilizadas en la construcción. Cada una de esas categorías queda representada por seis muestras, excepto las de arcillas de construcción de las que se obtuvieron como se expresa en la tabla siguiente:

\begin{tabular}{|ll|}
\hline CATEGORIA & $\mathrm{N}^{\circ}$ DE MUESTRA \\
\hline Escobillada & $1,9,11,12,22,23$ \\
Gris & $2,7,17,24,25,26$ \\
Castrena & $3,4,5,6,10,13$ \\
A Torno & $18,27,28,29,30,31$ \\
Molde de Situla & $14,15,16,32,33,34$ \\
Arcilla & $8,19,20,21$ \\
\hline
\end{tabular}

Tabla 1.-Categorias arqueológicas y relación de muestras.

\section{a) Fluorescencia de Rayos $X^{2}$}

El método de análisis utilizado para obtener la composición geoquímica de las cerámicas y arcillas fue el de Fluorescencia de Rayos X (FRX) (Dupont, 1977; Kardos et al., 1985). Los análisis se efectuaron con un espectrómetro secuencial Siemens SRS 300 con tubo de rodio

2 Todos los análisis se llevaron a cabo en el Centro de Ciencias Medioambientales del CSIC. de ventana equipado con un microordenador PdP 11/23. El método de preparación de muestras fue el de la Perla de Borax.

Con este método se determinaron los óxidos mayoritarios y minoritarios de cada una de las muestras expresados en tantos por ciento: $\mathrm{Al}_{2} \mathrm{O}_{3}, \mathrm{CaO}, \mathrm{SiO}_{2}, \mathrm{P}_{2} \mathrm{O}_{5}, \mathrm{TiO}_{2}, \mathrm{MnO}, \mathrm{Fe}_{2} \mathrm{O}_{3}$, $\mathrm{Na}_{2} \mathrm{O}, \mathrm{MgO}$ y $\mathrm{K}_{2} \mathrm{O}$, como podemos ver en la Tabla 2.

\section{b) Tratamiento de los datos}

Para el tratamiento de los datos escogimos, dentro del conjunto de métodos de análisis multivariante, el análisis discriminante. Este es un método de cálculo estadistico que permite estudiar la existencia de diferencias entre grupos, en este caso de categorías cerámicas, definidos mediante variables cuantitativas, a partir de los elementos químicos de las cerámicas. Nos permite entonces determinar las probabilidades de pertenencia de una cerámica a grupos arqueológicos establecidos previamente (Picón, 1984; Esquivel, 1990).

La utilización del análisis discriminante nos permitió observar si existe una correspondencia entre la geoquimica de las cerámicas estudiadas (Tabla 2) y las diferentes categorias arqueológicas consideradas (Tabla 1).

Utilizando como factor de clasificación para el análisis discriminante las categorías cerámicas observamos:

1. Una discriminación muy clara de las agrupaciones arcilla sin cocer $\left(n{ }^{\circ} 4\right)$ y arcilla cocida $\left(\mathrm{n}^{\circ}{ }^{\circ} 7\right)$ del resto de las categorias utilizadas en el análisis (Gráfico 1, Tabla 3).

2. El grupo de cerámicas hechas a torno, identificadas con el n. ${ }^{\circ} 6$, se aparta, excepto en un caso, del resto del material.

3. Las cerámicas que hemos denominado castreñas $\left(\mathrm{n} .^{\circ} 2\right)$ se discriminan del resto del material constituido por los cuatro grupos de cerámicas que no presentaron caracteristicas geoquímicas bien definidas.

Con la intención de conseguir una discriminación más real de la materia según las categorias arqueológicas, se aplicó un nuevo factor de clasificación para el análisis discriminante de tal forma que: El grupo 1 incluye las catego- 


\begin{tabular}{|c|c|c|c|c|c|c|c|c|c|c|}
\hline 1 & 15.25 & 0.77 & 60.35 & 1.88 & 0.87 & 0.06 & 6.30 & 1.35 & 1.54 & 2.74 \\
\hline 2 & 14.80 & 0.91 & 60.02 & 1.52 & 0.91 & 0.09 & 6.21 & 1.13 & 1.86 & 2.81 \\
\hline 3 & 13.34 & 1.00 & 57.75 & 3.05 & 0.72 & 0.05 & 5.30 & 1.72 & 1.19 & 2.95 \\
\hline 4 & 16.85 & 0.64 & 54.52 & 3.62 & 0.73 & 0.03 & 6.44 & 1.86 & 0.72 & 3.17 \\
\hline 5 & 16.09 & 1.19 & 57.84 & 1.55 & 0.61 & 0.04 & 5.46 & 1.56 & 1.37 & 3.76 \\
\hline 6 & 17.27 & 0.73 & 54.83 & 2.39 & 0.76 & 0.02 & 5.61 & 1.91 & 0.65 & 3.09 \\
\hline 7 & 16.01 & 0.76 & 61.30 & 1.35 & 0.97 & 0.11 & 6.65 & 1.54 & 2.28 & 2.96 \\
\hline 8 & 17.39 & 0.49 & 56,82 & 0.41 & 1.01 & 0.10 & 7.89 & 1.16 & 0.88 & 3.01 \\
\hline 9 & 12.87 & 0.71 & 55.23 & 3.27 & 0.90 & 0.08 & 6.03 & 1.46 & 1.47 & 2.54 \\
\hline 10 & 15.59 & 0.65 & 49.27 & 0.55 & 0.74 & 0.04 & 7.68 & 1.28 & 1.76 & 3.19 \\
\hline 11 & 12.44 & 0.88 & 56.01 & 0.98 & 0.74 & 0.12 & 5.44 & 1.41 & 1.44 & 2.88 \\
\hline 12 & 13.94 & 0.67 & 59.60 & 1.62 & 0.93 & 0.08 & 6.42 & 1.52 & 1.99 & 2.98 \\
\hline 13 & 13.32 & 0.70 & 58.56 & 2.17 & 0.74 & 0.05 & 5.18 & 1.96 & 1.42 & 3.13 \\
\hline 14 & 11.40 & 0.22 & 59.27 & 0.32 & 0.76 & 0.05 & 2.36 & 2.36 & 1.34 & 2.50 \\
\hline 15 & 13.27 & 1.29 & 54.98 & 1.58 & 0.77 & 0.16 & 6.84 & 1.87 & 1.41 & 2.83 \\
\hline 16 & 15.41 & 1.24 & 54.98 & 1.94 & 0.83 & 0.07 & 6.46 & 2.11 & 1.67 & 3.55 \\
\hline 17 & 14.02 & 0.92 & 54.69 & 2.46 & 0.92 & 0.12 & 6.39 & 1.24 & 1.92 & 2.86 \\
\hline 18 & 18.00 & 1.21 & 50.52 & 2.97 & 0.78 & 0.53 & 7.47 & 1.00 & 1.17 & 2.44 \\
\hline 19 & 18.05 & 0.44 & 53.22 & 0.28 & 0.94 & 0.05 & 7.46 & 1.31 & 0.81 & 3.32 \\
\hline 20 & 17.82 & 0.27 & 54.20 & 0.17 & 1.01 & 0.06 & 8.00 & 1.22 & 0.74 & 3.39 \\
\hline 21 & 17.27 & 0.04 & 46.73 & 0.29 & 0.94 & 0.04 & 7.20 & 1.16 & 0.39 & 2.62 \\
\hline 22 & 15.69 & 0.94 & 58.67 & 3.44 & 0.91 & 0.14 & 6.19 & 0.56 & 1.66 & 3.02 \\
\hline 23 & 14.36 & 1.00 & 57.70 & 3.31 & 0.88 & 0.12 & 5.99 & 0.90 & 1.54 & 2.96 \\
\hline 24 & 16.50 & 0.98 & 61.09 & 1.65 & 0.96 & 0.10 & 6.50 & 0.70 & 2.34 & 3.24 \\
\hline 25 & 17.85 & 1.06 & 63.95 & 1.16 & 0.96 & 0.10 & 6.52 & 0.25 & 2.20 & 3.68 \\
\hline 26 & 14.83 & 1.01 & 59.46 & 1.67 & 0.95 & 0.10 & 6.35 & 0.26 & 2.26 & 3.01 \\
\hline 27 & 19.49 & 1.46 & 59.59 & 0.86 & 0.75 & 0.04 & 7.10 & 0.39 & 1.65 & 3.42 \\
\hline 28 & 16.41 & 0.72 & 57.94 & 3.00 & 1.00 & 0.10 & 6.81 & 0.49 & 1.92 & 2.40 \\
\hline 29 & 21.39 & 3.59 & 49.64 & 2.10 & 0.80 & 0.03 & 7.05 & 0.28 & 2.22 & 4.17 \\
\hline 30 & 20.98 & 1.37 & 56.56 & 2.58 & 0.75 & 0.04 & 7.16 & 0.91 & 1.37 & 3.52 \\
\hline 31 & 15.11 & 0.91 & 61.81 & 1.04 & 0.94 & 0.04 & 6.98 & 0.44 & 1.68 & 2.84 \\
\hline 32 & 13.16 & 0.87 & 60.74 & 1.70 & 0.79 & 0.07 & 5.42 & 1.84 & 1.42 & 2.75 \\
\hline 33 & 15.97 & 1.17 & 56.61 & 1.36 & 0.88 & 0.08 & 6.61 & 1.71 & 2.13 & 3.65 \\
\hline 34 & 15.15 & 1.32 & 54.72 & 1.42 & 0.85 & 0.07 & 6.36 & 0.66 & 2.33 & 3.54 \\
\hline
\end{tabular}

Tabla 2.-Resultados obtenidos por Fluorescencia de Rayos X.

Agrupación

cat. arqu.

\section{Real Agrupación Teórica} $\begin{array}{ccccccc}1 & 2 & 3 & 4 & 5 & 6 & 7 \\ \text { escobillas } & \text { castreñas } & \text { grises } & \text { arc. sin coc. } & \text { sítulas } & \text { a torno } & \text { arc.coc. }\end{array}$

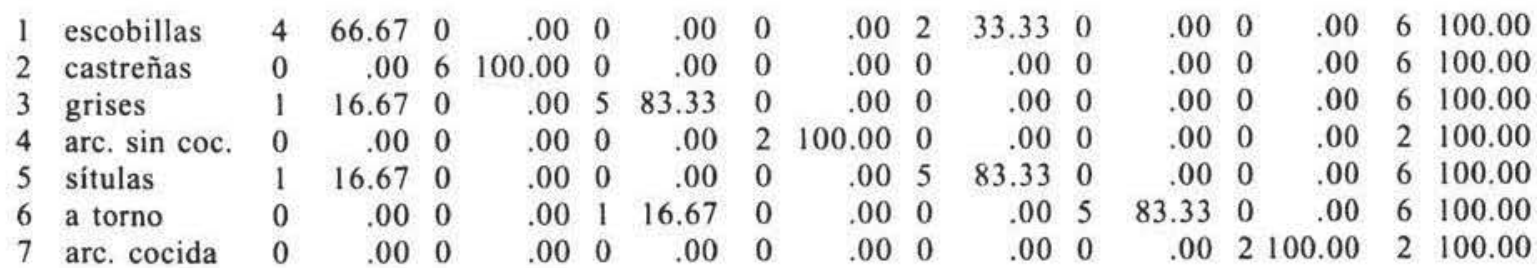

Tabla 3.-Análisis Discriminante. Clasificación de categorías arqueológicas según los \% de óxidos. Datos correspondientes al Gráfico 1. 
rias denominadas escobillado y gris; el grupo 2 las castreñas; el grupo 3 las cuatro muestras de arcilla cocida y sin cocer; el grupo 4 los moldes de situlas; el grupo 5 los fragmentos de cerámica a torno. Los resultados de este análisis fueron muy similares a los obtenidos con el anterior factor de clasificación.

El segundo caso se llevó a cabo con agrupaciones distintas del material, quedando las categorias arqueológicas escobillada, gris y castreña en un único grupo, el $\mathrm{n} .{ }^{\circ} \mathrm{I}, \operatorname{los}$ moldes de situlas en otro identificado con el $\mathrm{n} .^{\circ} 2$, las cerámicas a torno en el 3 y las arcillas en el 4. En este caso como podemos observar en el Gráfico 2, Tabla 4:

1. Una clara discriminación de las arcillas por una parte y de las cerámicas a torno por otra, del resto del material.

2. Una cierta asociación del resto de las categorías.

Por último se realizó un tercer análisis discriminante sin utilizar la categoria a torno. De esta forma las cerámicas escobilladas se agruparon en el número I, las castreñas en el 2, las grises en el 3, los moldes de situlas en el 4 y las arcillas en el 5. En este caso los resultados, reflejados en el Gráfico 3, Tabla 5 . nos permiten discriminar claramente las arcillas $8 \mathrm{n}^{\circ}{ }^{\circ}$ ) y las cerámicas denominadas castreñas $\left(\mathrm{n}^{\circ}{ }^{\circ}\right)$, de las grises $\left(\mathrm{n} .^{\circ} 3\right)$, escobilladas (n. $\left.{ }^{\circ} 1\right)$ y moldes de situlas ( $\mathrm{n}^{\circ} 4$ ), quedando estas dos últimas categorias dentro de un mismo grupo.

\section{DISCUSIÓN Y CONCLUSIONES}

Este trabajo sobre las composiciones geoquímicas de las cerámicas de El Castrelin de San Juan de Paluezas, continúa una serie de estudios mineralógicos y geoquímicos que sobre conjuntos cerámicos venimos, algunos de nosotros, realizando desde hace tiempo (Galván Martínez, 1986; Galván Garcia y Galván Martínez, 1987, 1988 y 1990). A raiz de las últimas investigaciones (Galván Martínez, 1991) com-

\section{Agrupación cat. arqu.}

$$
\begin{aligned}
& 1 \\
& \text { esc., gris, castr. }
\end{aligned}
$$

$\begin{array}{lrr}\text { esc. gris, castr. } & 15 & 83.33 \\ \text { situlas } & 1 & 16.67 \\ \text { a torno } & 0 & .00 \\ \text { arcillas } & 0 & .00\end{array}$

Real Agrupación Teórica

\begin{tabular}{|c|c|c|c|c|c|c|}
\hline $\begin{array}{c}2 \\
\text { situlas }\end{array}$ & \multicolumn{2}{|c|}{$\begin{array}{c}3 \\
\text { a torno }\end{array}$} & \multicolumn{2}{|c|}{$\begin{array}{c}4 \\
\text { arcillas }\end{array}$} & \multicolumn{2}{|c|}{ TOTAL } \\
\hline 16.67 & 0 & .00 & 0 & .00 & 18 & 100.00 \\
\hline 83.33 & 0 & .00 & 0 & .00 & 6 & 100.00 \\
\hline .00 & 6 & 100.00 & 0 & .00 & 6 & 100.00 \\
\hline .00 & 0 & .00 & 4 & 100.00 & 4 & 100.00 \\
\hline
\end{tabular}

Tabla 4.-Análisis Discriminante. Clasificación de categorias arqueológicas según los \% de óxidos Datos correspondientes al Gráfico 2.

\section{Agrupación} cat. arqu.

$\begin{array}{ll}1 & \text { escobilladas } \\ 2 & \text { castreñas } \\ 3 & \text { grises } \\ 4 & \text { situlas } \\ 5 & \text { arcillas }\end{array}$

Real Agrupación Teórica

\begin{tabular}{|c|c|c|c|c|c|c|c|c|c|c|c|}
\hline \multicolumn{2}{|c|}{$\frac{1}{\text { escobilladas }}$} & \multicolumn{2}{|c|}{$\begin{array}{c}2 \\
\text { castreñas }\end{array}$} & \multicolumn{2}{|c|}{$\stackrel{3}{\text { grises }}$} & \multicolumn{2}{|c|}{$\begin{array}{c}4 \\
\text { situlas }\end{array}$} & \multicolumn{2}{|c|}{$\begin{array}{c}5 \\
\text { arcillas }\end{array}$} & \multicolumn{2}{|c|}{ TOTAL } \\
\hline 6 & 100.00 & 0 & .00 & 0 & .00 & 0 & .00 & 0 & .00 & 6. & 100.00 \\
\hline 0 & .00 & 6 & 100.00 & 0 & .00 & 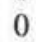 & .00 & & .00 & 6 & 100.00 \\
\hline 0 & .00 & 0 & .00 & 6 & 100.00 & 0 & .00 & & .00 & 6 & 100.00 \\
\hline 0 & .00 & 0 & .00 & 0 & .00 & 6 & 100.00 & 0 & .00 & 6 & 100.00 \\
\hline 0 & .00 & 0 & .00 & 0 & .00 & 0 & .00 & 4 & 100.00 & 4 & 100.00 \\
\hline
\end{tabular}

Tabla 5.-Análisis Discriminante. Clasificación de categorias arqueológicas según los \% de óxidos. Datos correspondientes al Gráfico 3. 
Gráfico 1.-El grupo número I corresponde a las cerámicas descritas como escobilladas. El 2 a las cerámicas denominadas castreñas. El 3 a las denominadas grises. El 4 a las muestras de arcilla sin cocer: arcilla de alzado y arcilla de pavimento. El 5 a los fragmentos de moldes de situlas. El 6 a los fragmentos de cerámica hecha a torno. El 7 a las muestras de arcilli cocida: fragmentos de pared de horno.

Gráfico 2.-Grupo 1, corresponde a las cerámicas descritas como escobillado, castreño y gris. Grupo 2, corresponde a los moldes de situlas. Grupo 3, corresponde a las cerámicas fabricadas a torno. Grupo 4 , corresponde a las cuatro muestras de arcillas.

Gráfico 3.-Grupo 1, corresponde a las cerámicas definidas con la categoria arqueológica de escobillado. Grupo 2, corresponde a las cerámicas definidas como castreñas. Grupo 3, corresponde a las cerámicas definidas como grises. Grupo 4, corresponde a los fragmentos de moldes de situlas. Grupo 5, corresponde a las muestras de arcilla.
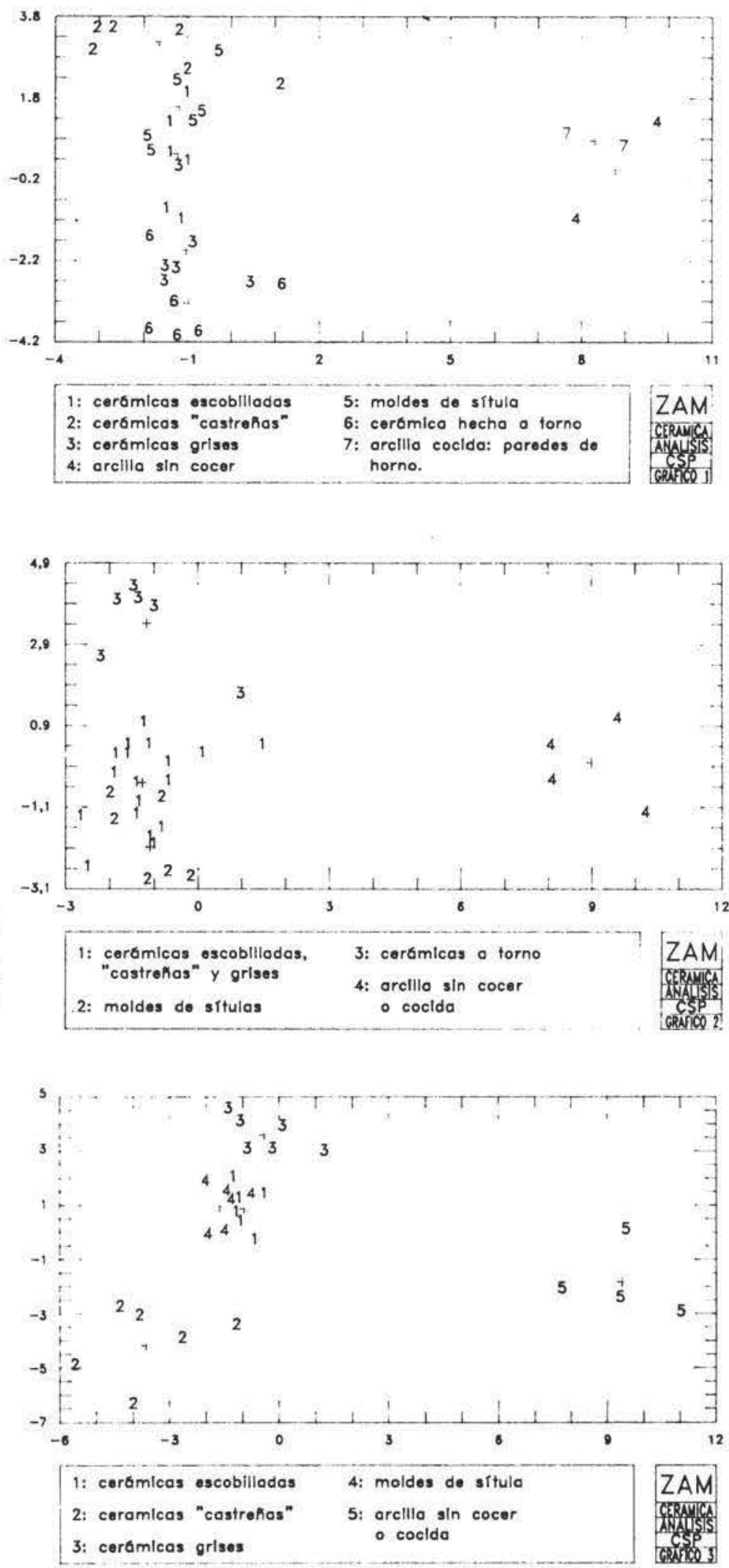
probamos que utilizando diversos métodos de análisis estadistico en el tratamiento de datos, en este caso de porcentajes de óxidos mayoritarios y minoritarios en las cerámicas, podemos llegar a resolver problemas arqueológicos relativos a la fabricación de las mismas, demostrando que las composiciones geoquimicas pueden corresponderse con algunas de las diferencias formales o funcionales.

Para ello aplicamos sistemáticamente el análisis discriminante hasta obtener la separación más adecuada del material según las categorias arqueológicas prefijadas de forma hipotética. Asi vemos en el caso que nos ocupa que algunas discriminaciones eran ya bastante nitidas desde los primeros análisis efectuados: las cerámicas a torno y las arcillas siempre aparecen separadas del resto del material cualquiera que sean los factores de discriminación (Gráfi$\cos 1$ y 2 ; Tablas 3 y 4). De igual forma los fragmentos de moldes de vitulas aparecen siempre asociados a la categoria castreña, la más común del yacimiento, como se muestra claramente en el Gráfico 3, Tabla 5.

De lo anterior se puede plantear, de acuerdo con nuestros objetivos, las siguientes cuestiones:

- La discriminación que tan patentemente afecta a las cerámicas a torno, parece estar indicándonos que las materias primas utilizadas para su fabricación no son las mismas que las que componen el resto del utillaje cerámico del yacimiento; como tampoco son las mismas las técnicas y el proceso de fabricación. Siendo su geoquimica tan poco equiparable a la de otros grupos cerámicos, la explicación parece estas en la posible procedencia de estas manufacturas a torno del comercio externo. No en vano representan solamente un mínimo porcentaje de la cerámica del yacimiento, en torno al $1 \%$ más concretamente, y se pueden asimilar a la cerámica cettibérica de los siglos II y 1 antes de nuestra era.

- Completamente diferentes son los resultados que afectan a los moldes cerámicos para fabricar sítulas. Su clara similaridad con la cerámica más común en el yacimiento - la más abundante y la que es general en los castros leoneses de la Segunda Edad del Hierro- nos está indicando, contra lo que podia esperarse, su fabricación local. Como ya dijimos anteriormente, este tipo de objeto tenia hasta el momento una conocida dispersión restringida a las áreas litorales gallega y portuguesa. Ahora, además de constatarse una interacción de ámbito geográfico más amplio entre las comunidades del Noroeste peninsular, podemos comprobar cómo una manufactura de especial significado y difícil producción se realiza en el propio poblado.

- Mucho menos clara es la respectiva discriminación de las cerámicas grises y escobilladas entre si y el grupo denominado castreño que sólo responde al análisis discriminante de forma débil y matizada. Sin embargo lo hacen (Gráficos 1 y 3; Tablas 3 y 5 ) en la medida suficiente como para que puedan ser tomadas en consideración ciertas diferencias premeditadas en su fabricación, aunque adquieran significado solamente cuando se tienen en cuenta otros factores como son sus matrices morfométricas, su funcionalidad o, como en el caso concreto de las grises, su escasa presencia y aspecto evolucionado dentro del conjunto cerámico del yacimiento. Es decir sin estar fabricadas con otra materia prima que las castreñas, se discriminan por una composición premeditadamente diferenciada, en una voluntad, como en el caso de los moldes de situlas, de alcanzar una determinada calidad en el resultado del producto. Aunque no podamos establecer el alcance de tales diferencias, queda claro que el método analítico utilizado se valida para el establecimiento de «clases» cerámicas, en un intento de superar las tipologías morfológicas al uso.

- Son también interesantes las diferencias significativas puestas de manifiesto entre las arcillas utilizadas en los materiales de construcción de algunos elementos del poblado: alzados, pavimentos de las construcciones e incluso construcciones de hornos y las utilizadas para la fabricación de las cerámicas. Circunstancia que nos informa sobre la utilización y transformación, es decir, la inversión de trabajo y tiempo, de los materiales disponibles en torno al poblado por sus ocupantes.

Como puede observarse, la finalidad de este trabajo, queda cumplida en la operatividad 
que como indicador arqueológico contienen los componentes mineralógicos y geoquímicos de los repertorios cerámicos de los yacimientos, asi como la metodología empleada, por más que sean susceptibles de mejora, tanto en el necesario planteamiento de los interrogantes más estrictamente arqueológicos, como en la interpretación de los resultados.

\section{BIBLIOGRAFÍA}

Carballo Arceo, L. X., 1983: Aportación al estudio de las situlas en el Occidente de la Península Ibérica. Cuadernos de Estudios Gallegos, XXXIV, 99:7-32.

Dupont, P., 1977: Recherches de Laboratoire sur les Céramiques Grecques Orientales Archaiques. Revue d'Archeometrie, 1:105-114.

Esquivel Guerrero, J. A., 1990: Un algoritmo de análisis discriminante múltiple aplicado al estudio de un complejo cerámico de la Edad del Bronce. Aplicaciones Informáticas en Arqueología. Reunión de 1990. Resúmenes de las Comunicaciones.

Fernández-Posse, M. D., y Sánchez-Palencia, F.-J., 1988: La Corona y el Castro de Corporales. II. Truchas (León). Prospecciones Arqueológicas en la Valderia y La Cabrera. E.A.E., 153. Madrid.

Galván García, J., y Galván Martínez, V., 1987: Estudio Mineralógico de Cerámicas procedentes de «El Llanete de Los Moros» (Montoro, Córdoba). E.A.E., 151, 270-278.

Galván García, J., y Galván Martínez, V., 1988:
Estudio Mineralógico de Cerámicas y ArciIlas procedentes de La Corona y El Castro de Corporales (León). E.A.E., 153, 249-250.

Galván Garcia, J., y Galván Martínez, V.. 1990: Estudio Mineralógico de algunos fragmentos de ('erámicas y Arcillas procedentes del yacimiento de Oxkintok. Oxkintok 3, 127 134.

Galván Martínez, V., 1986: Análisis de Pastas Cerámicas. Huelva Arqueológica 8: 277-331.

Galván Martinez. V., 1991: Análisis Mineralógico y Geoquímico de Cerámicas procedentes del SE Peninsular. Tesis Doctoral inédita. Universidad Autónoma de Madrid.

Kardos, J.; Zimmer, K.; Kriston, L.; Morozova, O.; Träger, T., y Jerem, E., 1985: Scientific Investigations of the Sopron-Krautacker Iron Age Pottery Workshop. Archeometry 27, 1:83-95.

Picón, M.: Le Traitement des Donnés d'Analyse. PACT, 10, 380-399.

Sánchez Palencia, F.-J., y Fernández-Posse, M. D., 1985: La Corona y El Castro de Corporales. I. Truchas (León). E.A.E., 141. Madrid.

Sánchez Palencia, F.-J., y Fernández-Posse, M. D., 1986: Hábitat y Urbanismo en La Corona de Corporales. Arqueologia Espacial, 9. Teruel.

Sánchez Palencia, F.-J., y Fernández-Posse, M. D.; Fernández Manzano, J.; Álvarez González, Y, y López González, L. F., 1990: La Zona Arqueológica de Las Médulas (198889). $A E \operatorname{sp} A, 63,249-264$. 